\title{
Study of the Best Checkpoint Interval in the Distributed Simulation System Based on Virtualization Technology
}

\author{
$\mathrm{GeYu}^{1, \mathrm{a}}$, Yang Yunfei ${ }^{1, \mathrm{~b}}$, Zhu Chengwen ${ }^{1, \mathrm{c}}$, Song Miao ${ }^{2, \mathrm{~d}}$, Cai Xiaoyan ${ }^{1, \mathrm{e}}$, and \\ Zheng Yan $^{1, \mathrm{f}}$ \\ ${ }^{1}$ Wuhan Mechanical Technology College, Wuhan Hubei, China \\ ${ }^{2}$ Military Representative Office of Beijing, Beijing, China \\ ageyu1982@126.com, bdaoke@163.com
}

\begin{abstract}
Keywords: Virtualization technology; Distributed simulation system; Rollback recovery; Fault tolerance; Checkpoint interval
\end{abstract}

\begin{abstract}
In order to keep the system's transfer, every once in a while, it was needed to grab a snapshot for the VM of all the nodes which were in the process of the distributed simulation system. It is very important to set the reasonable checkpoint interval for optimizing the system's average utilization rate. By establishing the mathematical model, The availability of distributed simulation system based on virtual technology is analyzed, and the solving equations for getting the biggest system availability is obtained, then the best checkpoint interval of the system fault tolerance could be obtained too, and the system fault tolerance of the simulation system was solved, which was so important to improve the effectiveness and credibility of the system.
\end{abstract}

\section{Introduction}

To solve the problem of its fault tolerance is so important to improve the effectiveness and credibility of the distributed simulation system[1] based on virtualization technology when which was running. Normally rollback recovery can be used to realize the fault tolerance system. In order to keep the system's transfer, every once in a while, it needed to grab a snapshot for the VM of all the nodes, These nodes could also be referred to as the checkpoints. Then all the simulation nodes can continue to run the simulation after its recovering from the recent checkpoint when the whole system could not run because of the hardware failures or power outages, etc.During the simulation task's runtime, to find a suitable checkpoint was the focus of the rollback recovery. It would seriously affect the performance and the throughput of the system if the checkpoint interval was set too closely, on the contrary, the failure process would be too high and the rollback recovery's cost would be big if the checkpoint interval was set too sparsely. So setting up reasonable checkpoint interval would partly optimize the average utilization rate of the system.

\section{The note of the distributed simulation system based on virtualization technology}

Using the virtualization technology, which can achieve the purpose of running multiple operating system at the same time, and the multiple programs could run in parallel in each operating system, and each operating system run in a virtual CPU or an virtual host, the great progress of the technology was mainly manifested in the virtual machine's related cost and which could support various operating system more widely.

For the distributed simulation system[2] based on virtual technology, all the works were done for the customer, to consider the needs of the users, quickly polymerized the simulation resource already existed in the operating system, and so it could achieve the development of personalized simulation application system which was driven by the user's needs.

In the virtual machine, the key components of the virtual machine management belong to all the physical node cluster could manage the virtual machine which was running in the distributed system, And to collect the state information of the simulation nodes which the virtual machine manager belonged to. The performance of the virtual machine and the simulation system which were running 
on the simulation nodes were directly influenced by the performance of the nodes. If the simulation nodes were determined then the checkpoint intervals would be defined too. The optimal checkpoint interval was one of the key problems to construct the distributed simulation system based on the virtualization technology.

\section{The analysis of the traditional model of the optimal checkpoint interval}

A.How to set the time parameter of the optimum checkpoint interval

1)The execution time of the simulation task The operation time refers to the simulation time which contains the process from the start to the completion of the simulation task, which was the task's operation time in theory, and in the operation process the fault tolerant measures were not used and there is no fault.

The time $\mathrm{T}$ is divided into $\mathrm{n}$ times, each time period is $\varepsilon \mathrm{i}$.

2) Set up the $C$ which was the costs of the checkpoints It needed to set up the checkpoints at the end of the $\mathrm{N}$ times which belong to the $\mathrm{t}$, and the $\mathrm{t}$ was the execution time of the simulation task. And the $\mathrm{c}$ is the overhead when the each checkpoint was set up.

3)Fault processing time - Td The Td was that takes to the checkpoint and recovery in the process of the time from the start to the completion of the tasks.

Then the total time of task execution was TW=T+Tr+Td.

Set up the number of the time intervals in the process of the simulation task's execution is $n(\varepsilon)$, so based on the set in the above, the execution time of the task which was in Trouble-free process was $\mathrm{T}=\varepsilon n(\varepsilon)$, it did not need to set up checkpoints in the last time interval because at that time the task would be going to the end. So the overhead of the checkpoint's setting up was $\mathrm{C}(\mathrm{n}(\varepsilon)-1)$.

The total time of each interval's execution should be the sum of the time interval of the checkpoint and the costs to set up checkpoints, which was the $\varepsilon+\mathrm{C}$.

Assume that the probability of the failure was the same in the process of each interval, then the fault processing time

$\mathrm{Td}=(\varepsilon+\mathrm{C}) \mathrm{N}(\varepsilon)$.

The $N(\varepsilon)$ was the interruption times of the fault. So it can be get that

$$
T_{W}(\varepsilon)=\varepsilon N(\varepsilon)+C(N(\varepsilon)-1)+\frac{1}{2}(\varepsilon+C) N(\varepsilon)
$$

B.Best checkpoint's numerical analysis in the system

Assume the system's fault obeys the Poisson distribution, and the fault model was $e^{\lambda \varepsilon}$, The task was fixed in the execution time of $\mathrm{T}$ without fault, The total time of the task was divided into $\mathrm{n}(\varepsilon)$ time intervals whose size was $\varepsilon$, then the expectation of the fault number is the product of the number of the divided segments and the failure probability of each segment. So that

$$
T_{W}(\varepsilon)=T+C\left(\frac{T}{\varepsilon}-1\right)+\frac{1}{2}(\varepsilon+C) \frac{T}{\varepsilon}\left(e^{\lambda \varepsilon}-1\right)
$$

The availability of A of the distributed simulation system should be the ratio of T to TW. That was

$$
A=\frac{T}{T+C\left(\frac{T}{\varepsilon}-1\right)+\frac{1}{2}(\varepsilon+C) \frac{T}{\varepsilon}\left(e^{\lambda \varepsilon}-1\right)}
$$

\section{Checkpoint interval model of the distributed simulation system based on virtualization technology}

In the distributed simulation system[3,4,5], when the error occurs which the simulation nodes were not in the running state, according to the space and time consistency strategy which was for 
high availability, through the checkpoint rollback technology, the system realized the fault-tolerant migration.

Assume that the number of physical nodes in distributed simulation system was $\mathrm{N}$, only considered the hardware failure such as the processor to stop response or collapse, and so on, which caused by the hardware's unstable or the interruption of power supply. And at the same time, it was assumed that the software and network was error-free. The time interval of the processor's twice errors obeyed the index distribution, and which the processor's failure was happened or not were independent of each other, the system occurs only at most two rolls back in a checkpoint interval time. Moreover, suppose that the checkpoint overhead c and the fault recovery's cost $\mathrm{R}$ were all the constants.

Set up the checkpoint interval as $\mathrm{T}$, that was, the simulation system performed a checkpoint every T time which was the system's propulsion time. The RTime refers to the actual cost time of the checkpoint interval.

The availability of the system

$$
\mathrm{A}=\mathrm{T} / \mathrm{R}_{\text {Time }}
$$

Based on the above model, when complete a checkpoint interval which was in three of the following: there was no fault in the system or there was an error in the system with one rollback recovery or there were two errors in the system with two rollback recoveries. The three conditions occur at a certain probability, the corresponding RTime each was also not identical. In the actual processing, Which used the RTime's expectations (average) to solve the system's availability. The best checkpoint interval requested was the t which could make the system availability A to obtain the maximum.

A. There was a normal advance of the System at the completion of a checkpoint interval, no error occurs

The time interval which was the time of the $\mathrm{N}$ physical nodes in the system error twice was greater than $\mathrm{T}+\mathrm{C}$.

Due to the processor's twice failure time interval obey the exponential distribution which was the continuous random variables. The distribution function was ${ }^{1-} e^{-\lambda t}(\mathrm{t}>0), \mathrm{RTime} 1=\mathrm{T}+\mathrm{C}$.

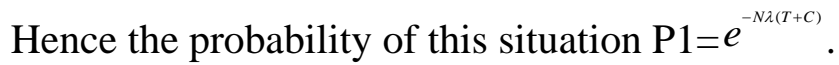

The checkpoint interval's execution was as shown in the figure1.

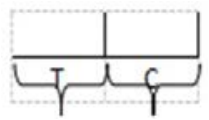

Figure 1 the checkpoint interval's execution if there was no error

B.Only one rollback recovery occurs in the system

In the $\mathrm{N}$ processors, only one broke down at $0 \sim \mathrm{T}$ time when the checkpoint interval was beginning[6,7]. Then the rollback recovery system began to run until which was completed. And within the time $t+2 \mathrm{R}+\mathrm{C}$, The rest of the $\mathrm{N}-1$ processors were not in down. In this case, $\mathrm{R}_{\mathrm{Time} 2}=\mathrm{T} 1+\mathrm{R}+\mathrm{T}+\mathrm{C}, \quad \mathrm{T} 1=\int_{0}^{T} t \lambda e^{-\lambda t} d t=(\mathrm{T}+1 / \lambda) e^{-\lambda T}+1 /($ The probability density of exponential distribution is $\left.\mathrm{f}(\mathrm{t})=\lambda e^{-\lambda t}(\mathrm{t}>0)\right)$. Due to the processor may be brook down at any time in the $0 \sim \mathrm{T}$ times, the $\mathrm{T} 1$ refers to the expectation. The probability of this happening was $\mathrm{P} 2=$ $A_{N}^{1}\left(1-e^{-\lambda T}\right) e^{-\lambda(2 T+R+C)(N-1)}$. The checkpoint interval execution process was shown in figure 2 .

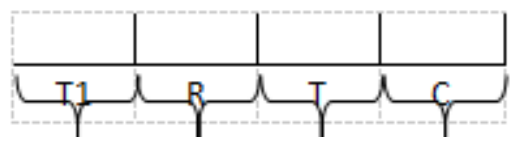

Figure 2 the checkpoint interval execution process if there was one error

C.The two rollback recoveries were happened in the system

In the $\mathrm{N}$ processors, only one broke down at $0 \sim \mathrm{T}$ time when the checkpoint interval was 
beginning. And then another broke down at $\mathrm{T}+\mathrm{R} \sim 2 \mathrm{~T}+\mathrm{R}$ time, And within the time $\mathrm{T}+2 \mathrm{R}+\mathrm{C}$, The rest of the $\mathrm{N}-2$ processors were not in down. In this case, $R_{\text {Time3 }}=2 T 1+2 R+T+C$, The probability of this happening was

$$
\mathrm{P} 3=\mathrm{A}^{2}\left(1-e^{-\lambda T}\right)\left\{e^{-\lambda(T+R)}-e^{-\lambda(2 T+R)}\right\} e^{-\lambda(3 T+2 R+C)(N-2)}
$$

The checkpoint interval execution process was shown in figure 3.

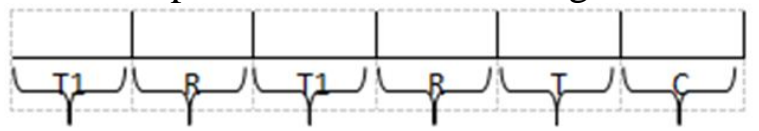

Figure 3 the checkpoint interval execution process if there were two errors

Because of the limit in this paper, which was there were only at most two rollback recoveries[8,9] in a checkpoint interval's running time. The expectation of the RTime was

$$
R_{\text {Time }}=\frac{P 1}{(P 1+P 2+P 3) * R_{\text {Time } 1}}+\frac{P 2}{(P 1+P 2+P 3) * R_{\text {Time } 2}}+\frac{P 3}{(P 1+P 2+P 3) * R_{\text {Time } 3}}
$$

To plug the formula (2) into the formula (1) then the A can be got, which was the system's availability.

$$
A=\frac{T(P 1+P 2+P 3)}{P 1 * R_{\text {Time } 1}+P 2 * R_{\text {Time } 2}+P 3 * R_{\text {Time } 3}}
$$

A was the function about $\mathrm{R}$ and $\mathrm{T}$ and $\mathrm{C}$, because in the actual in the distributed simulation system the $\mathrm{C}$ and $\mathrm{R}$ was known, So the function can be got about the $\mathrm{A}$ and $\mathrm{T}$. Obviously, when the availability of the system is the biggest, the corresponding checkpoint interval was the best checkpoint interval. Then took the derivative of the formula (3), Make the $\frac{d A}{d T}=0$, the $\mathrm{T}$ which was solved was the best checkpoint interval. The optimal checkpoint interval of a simulation task can be obtained by solving the formula. Because $\frac{d^{2} A}{d T^{2}}<0$, So under the given condition, the system availability has the only one great value.

\section{The test data}

A set of assumptions of the test data was used to analyze and verify the influence to the availability of the system if the different inspection interval was used. And all the time units were the s. The running time of the simulation task in theory was $5040 \mathrm{~s}$, the number of the simulation node is 100 , Checkpoint's cost was set up for 2 s, the availability which was got when the $\lambda$ was set to different values was shown in the table below.

\begin{tabular}{|c|c|c|}
\hline The checkpoint interval & The best availability & $\lambda$ \\
\hline $50.4 \mathrm{~s}$ & $82 \%$ & 0.00005 \\
\hline $42 \mathrm{~s}$ & $81.4 \%$ & 0.00007 \\
\hline $38.7 \mathrm{~s}$ & $81 \%$ & 0.00009 \\
\hline
\end{tabular}

Table1 the results of numerical analysis

The checkpoint interval effect the system's availability so more, when the checkpoint interval was too small, then the system need to set the check point frequently, Each of the check point settings would have certain costs, thus reduced the availability of the system; but if the checkpoint interval was too large, Once a failure occurs, the system must to be rollback to the checkpoint on the saved state, this happened a lot of losses, but also reduced the availability of the system.

\section{Conclusions}

The automatic test system has been applied in engineering practice, it use the standardization and modularization and open architecture, so the system can complete one tank's all system's detection and fault diagnosis and using the least physical resources, it is safe and reliable operation. The 
prominent characteristics of the automatic test system are the reuse of the platform's physical resources, and the test program has nothing to do with the hardware, so as to realize the general of the underlying equipment, swaps, guarantee the consistency of the test. In the process of one tank's works, if it appears some abnormal phenomenon, the automatic test system can quickly judge the fault's components, and it is a reliable analysis platform to determine the failure parts or failure unit circuit.

\section{References}

[1] LIU Yun-sheng, ZHANG Chuan-fu, ZHANG Tong. Socket Migration in Distributed Simulation System. Journal of System Simulation, National University of Defense Technology,2006.

[2] LI Bohu, CHAI Xudong, HOU Baocun, Networked Modeling \& Simulation Platform Based on Concept of Cloud Computing-Cloud Simulation Platform, Beijing Simulation Center,2009.

[3] Shi Rui , Zuo Decheng, Zhang Zhan. Fault Tolerant Topology Control in Mobile Ad Hoc Network, Journal of Beijing University of Posts and Telecommunications,2005.

[4] ZHANG Binbin,LUO Yingwei,WANG Xiaolin. Whole-System Live Migration Mechanism for Virtual Machines, ACTA ELECTRONICA SINICA,2009.

[5] Li Yunfa,Xu Xiang hua,Wan Jian. Load migration based resource schedul ing mechanismin virtual machine, Huazhong Univ. of Sci. \& Tech. (Natural Science Edition),2009.09

[6] E Manetho,et al.A survey of rollback recovery protocols inmessage passing systems[J].ACM Computing Surveys,2002,33(3):375-408.

[7] Y Ling,et al.A variational calculus approach to optimal checkpoint placement [J].IEEE Trans,2001,C-50(7):699 -707.

[8] Z. Li, W. Cai, S. J. Turner, K. Pan. A Replication Structure for Efficient and Fault-

[9] tolerant Parallel and Distributed Simulate[C]. Proc. of Spring Simulation MulticonferenceSpringSim, 2010:1-10 